\title{
miR-7a Targets Insulin Receptor Substrate-2 Gene and Suppresses Viability and Invasion of Cells in Diabetic Retinopathy Mice via PI3K-Akt-VEGF Pathway
}

This article was published in the following Dove Press journal:

Diabetes, Metabolic Syndrome and Obesity: Targets and Therapy

\author{
Zhenyu Ji ${ }^{1}$ \\ Jinyuan Luo' \\ Ting $\mathrm{Su}^{2}$ \\ Changzheng Chen' \\ Yu Su (D) \\ 'Department of Ophthalmology, Renmin \\ Hospital of Wuhan University, Wuhan, \\ Hubei, 430060, People's Republic of \\ China; ${ }^{2}$ Eye Institute of Xiamen \\ University, Xiamen University, Xiamen, \\ Fujian, 36II02, People's Republic of \\ China
}

Introduction: Diabetic retinopathy (DR) is one of the major leading causes for vision loss globally. Current study illustrates the role of miR-7a in DR.

Material and Methods: Retinal pericytes (RPs) and Endothelial cells (ECs) were isolated from mouse model of DR. qRT-PCR was done for expression of miR-7a and target gene mRNA, Western blot for protein expression. Identification of miR-7a target gene was done by TargetScan and Luciferase assay. Cell viability and invasion was done by MTT and Transwell chamber assay.

Results: The expression of miR-7a was down-regulated whereas level of IRS-2 was unregulated in isolated RPs and ECs. Luciferase assay suggested correlation between miR7a and IRS-2, over-expression of miR-7a using a mimic resulted in suppression in viability and invasion capacity of RPs and ECs and inhibited the protein levels of PI3K/Akt cascade and IRS-2, and however the inhibitor reversed them respectively. Transfection of siRNA targeting IRS-2 caused alteration in miR-7a mediated changes in ECs suggesting that miR-7a may decrease angiogenesis in DR by inhibiting the levels of IRS-2.

Conclusion: miR-7a suppresses PI3K/Akt cascade via targeting IRS-2, thus decreasing the viability and invasion capacity of RPs and ECs, suggesting an interesting treatment target for DR.

Keywords: miR-7a, diabetic retinopathy; DR, IRS-2, PI3K/Akt, endothelial cells; ECs, retinal pericytes; RPs

\section{Introduction}

Diabetic retinopathy (DR) is identified to be major causative factor leading to impaired vision and blindness in diabetic adults globally. ${ }^{1}$ It is one of the diabetic complications leading to early loss of vision. ${ }^{2}$ Thickening of basement membrane and loss of capillary pericytes are the major events which further lead to proliferation of endothelial membrane and at last angiogenesis. ${ }^{2}$ Vascular endothelial growth factor (VEGF) is major contributor in developing proliferative DR and macular edema. ${ }^{3}$ Targeting VEGF seems to be logical approach in treating DR, but suffers disadvantage of producing some systemic adverse effects. ${ }^{3}$ Hence, developing new therapies for treating DR are needed by studying and empathizing the involved mechanisms and pathways in its development and progression. Insulin receptor substrate-2 (IRS-2) is member of the IRS family and is an important molecule in
Department of Ophthalmology, Renmin Hospital of Wuhan University, Wuhan, Hubei, 430060, People's Republic of China $\mathrm{Tel} / \mathrm{Fax}+86-2788041911$

Email sy_daisy1206@I63.com
Diabetes, Metabolic Syndrome and Obesity: Targets and Therapy 2021:14 719-728 
insulin signaling along with IRS-1. Phosphoinositide 3-kinase (PI3K) and Akt pathway are identified to be the important pathway linking the IRS proteins to metabolic action of insulin. ${ }^{4}$ IRS-2 is reported to be the chief mediator of insulin-dependent mitogenesis and is responsible for regulation of glucose metabolism in cells, also it has been implicated in regulation of GLUT4 protein responsible for uptake of glucose. ${ }^{5}$ The angiogenic factor VEGF is reported to be involved in maintaining pathological angiogenesis in DR and vascular homeostasis, also treatment of VEGF is beneficial in DR. ${ }^{6,7}$ A previous study suggested that triggering of PI3K/Akt cascade leads to increased expression of VEGF by directly regulating the VEGF promoter. $^{8}$ In addition to this, long term over-expression of VEGF is regulated by activation of PI3K/Akt pathway. ${ }^{9}$ All the literature suggests that the IRS-2/PI3K/Akt/VEGF cascade could be a potential target for treating and preventing DR.

Diabetes is a disorder associated with pathological as well as vascular abnormalities. ${ }^{10}$ The mortality of cell types such as vascular smooth muscle, endothelial cells (ECs) and capillary pericytes is linked with poor formation of arteries and loss of endometrial function. ${ }^{11-14}$ Insulin is reported to stimulate number of pathways in endometrial cells. ${ }^{9}$ In addition to this, insulin plays a crucial role in triggering IRS/PI3K/Akt cascade. ${ }^{15}$ All these studies demonstrate the importance of IRS/PI3K/Akt axis and also suggest that that any regulatory event in this axis can affect process of angiogenesis in DR.

miRNAs (miRs) have emerged as non-coding RNAs, small and highly conserved which bind to target genes and regulate their expression. ${ }^{16}$ miR-7a is an conserved miRNA mainly involved in development of pancreas. ${ }^{17}$ miR-7a has been reported to modulate the expression of $\mathrm{VEGF}^{18}$ and matrix metalloproteinases, ${ }^{19}$ both the factors have critical role in development of DR. ${ }^{20}$ Also miR-7a has been found to be down-regulated in certain cancers and also regulate multiple signaling pathways such as phosphoinositide 3-kinase/protein kinase B (PI3K/AKT) and $\mathrm{RAF} /$ mitogen-activated protein kinase/extracellular signal-regulated kinase (RAF/MEK/ERK). ${ }^{21,22}$

In the present work, we studied the function of miR-7a in correlation to DR. We evidenced that, level of miR-7a was decreased significantly in Endometrial cells (ECs) and retinal pericytes (RPs) when compared to that in healthy group. We also found that this down-regulation of miR-7a caused over-expression of IRS-2 which in turn regulates the PI3K/Akt/VEGF cascade.

\section{Materials and Methods Confirmation of Animal Model of DR and Cell Culture}

Earlier the reports have studied and confirmed the interaction of ECs and Pericytes in the walls of blood vessels regulates the process of vessel formation their remodeling and stabilization, ${ }^{2}$ hence in the present work we selected RPs and ERs from mice model of DR. For the present study the mice were supplied by the animal center of Renmin Hospital of Wuhan University, Wuhan, Hubei, China. All the experiments were approved by the ethical review board of Renmin Hospital of Wuhan University, Wuhan, Hubei, China (Approval number 1341DR). The experiments were in accordance to Chinese National Guidelines (GB/T 35,892-20,181) for the ethical review of laboratory animal welfare People's Republic of China drafted by National Research Institute for Health; National Institutes for Food and Drug Control; Beijing Cancer Hospital; Sanofi (China) Investment Co., Ltd.; Institute of Laboratory Animal Sciences Chinese Academy of Medical Sciences Laboratory Animal Centre, Peking University; the Welfare and Ethics Committee of the Chinese Association for Laboratory Animal Sciences (CALAS). The animals were maintained at $25^{\circ} \mathrm{C}$ with $50 \%$ relative humidity and were exposed to 12 hour dark and light cycle and were provided free access to water and pellet diet ad libitum. For inducing diabetes we selected male C57/BL6 mice $(\mathrm{n}=30)$ (genetically modified mice) aging 12 weeks and weighing between 20-22 g, the mice were given daily injection of Streptozotocin $(45 \mathrm{mg} / \mathrm{kg}$ solubilized in citrate buffer $\mathrm{pH} 4.5$ ) for 5 days. The control mice $(n=10)$ were injected with buffered saline. After 7 days injecting the mice with streptozotocin, blood glucose levels were measured using glucose estimation kit following the supplied instructions (Sigma Aldrich USA) and mice showing fasting blood glucose levels of $200 \mathrm{mg} / \mathrm{dl}$ were graded diabetic and were used in the study. The mice model of DR was established as reported earlier. ${ }^{20}$ The RPs and ECs were isolated from the DR mice as described earlier ${ }^{23,24}$ and were validated by the microbiology department of our institute.The isolated RPs and ERs were maintained in minimum essential media (MEM) (ThermoFisher USA) which was added with Fetal bovine serum (FBS) 10\% (ThermoFisher USA), Penicillin (100 $\mathrm{U} / \mathrm{mL})$ and Streptomycin $(100 \mathrm{mg} / \mathrm{mL})$ (SigmaAldrich USA) the humidity was $85 \%$. The cells were maintained 
at $25^{\circ} \mathrm{C}$ under controlled condition of humidity and $5 \%$ carbon dioxide.

\section{qRT-PCR Analysis}

The qRT-PCR analysis was performed on Applied Biosystems ${ }^{\circledR}$ 7900HT Fast Real-Time PCR System using TaqMan MicroRNA assays (Thermo Fisher USA), the total RNA (200 ng) was isolated using TRIzol reagent (Invitrogen, Thermo Fisher USA). The isolated RNA was submitted for reverse transcription to cDNA with the help of Taqman miRNA reverse transcription kit (Thermo Fisher USA) following supplied instructions. The resultant cDNA was amplified with the help of Taqman miR-7a miRNA assay (Thermo Fisher USA) by PCR using the primers for miR-7a: forward 5'- TAGGATCCTGCTGCCAAAACCACCACTTGT-3'and reverse 5'- GCGAATTCTGCAGGGTTAAGAAAATGAA ACTGGA- $3^{\prime}$. The resultant mixture was maintained at $95^{\circ} \mathrm{C}$ for 10 minutes and then 40 cycles as $95^{\circ} \mathrm{C}$ for 10 seconds and $60^{\circ} \mathrm{C}$ for 60 seconds. The expression miR-7a was normalized against U6 mRNA and gene mRNA compared against Actin as loading control.

\section{Western Blot Analysis}

The isolated cells $\left(1 \times 10^{6}\right)$ were lysed with the help of lysis buffer (ThermoFisher USA). The total protein content was analyzed by protein estimation kit (Bio-Rad Lab. USA). About $30 \mathrm{mg}$ of total protein was loaded and separated on SDS-PAGE (10\%) and then transferred to nitrocellulose membranes. The membranes were blocked with $0.5 \%$ Triton-X-100 in Tris buffered saline along with 5\% nonfat milk at $4^{\circ} \mathrm{C}$ for 12 hours and incubated with primary antibodies of IRS-2 (1:500), PI3K (1:500), VEGF (1:500), Akt (1:500) and actin (1:500).All the antibodies were bought from Santa Cruz Biotech. USA. The membranes were rinsed with tris-buffered saline and incubated along with horseradish peroxidase conjugated $\mathrm{II}^{\mathrm{ry}}$ antibody (1:5000) for 90 minutes at $25^{\circ} \mathrm{C}$. After incubation, the membranes were rinsed with tris-buffered saline. The densitometric analysis of bands was performed using iBright Western blot imaging system (ThermoFisher USA). The expression of proteins was normalized against Actin which was loading control with the help of Image plus software.

\section{Transfection of Cells}

The murine isolated ECs and RPs $\left(1 \times 10^{6}\right)$ were transfected with recombinant plasmids and miR-7a mimic or mimic control (200 pmol) and miR-7a inhibitor or inhibitor control $(200 \mathrm{pmol})$. The process of transfection was carried by nonviral technique using Nucleofector II (Amaxa biosystems, BiocompareUSA) following the supplied instructions. For studying the IRS-2 involvement, HEK293 genomic DNA was opted as PCR template, expressing vector was modulated and inserted with amplified DNA fragment encoded for miR-7a pre-miR as reported earlier. ${ }^{25}$ After transfection the cells were maintained at $37^{\circ} \mathrm{C}$ for 5 hours and used for the experiments.

\section{Luciferase Assay}

Bioinformatics analysis was performed for identifying potential binding site of miR-7a on target genes using TargetScan. For confirming the results of bioinformatics analysis Luciferase assay was done.The DNA fragment of the IRS-2 3'-UTRhaving the predicted target site of miR-7a (IRS-2 wild type, WT) was cloned in the pGL3 luciferase vector (Promega Corp. USA). For generating mutated 3'UTR pGL3 luciferase vector the binding sites of miR-7a (IRS-2 mutant, MUT) were replaced with $4 \mathrm{nt}$ fragments. The luciferase vectors bearing mutated or mutant IRS-2 3'UTR were then co-transfected with miR-7a mimic or mimic control, miR-7a inhibitor or inhibitor control in the ECs cells with the help of Lipofectamine ${ }^{\mathbb{R}}$ MessengerMAX ${ }^{\mathrm{TM}}$ transfecting reagent (ThermoFisher USA). The Luciferase Reporter Gene detection kit (Sigma Aldrich USA) following the provided instructions.

\section{Cell Viability MTT Assay}

The viability of ECs and RPs was analyzed by performing 3-(4,5-dimethylthiazol-2yl)-2,5-diphenyltetrazolium bromide (MTT) assay. For MTT assay, the cells were submitted for seeding in 96 well plates with each well having $2 \times 10^{3}$ cells incubated in MEM media for time ranging between 24 to 120 hours. After incubation, the MEM media was rinsed off and was replaced with MTT (Sigma Aldrich USA) loaded fresh media $(5 \mathrm{mg} / \mathrm{mL})$, the cells were incubated with MTT for 5 hours. The formed Formazan crystals were solubilized using Dimethyl sulfoxide and the optical density was recorded at $490 \mathrm{~nm}$ with the help of ELISA reader after every 24 hours.

\section{Transwell Chamber Cell Invasion Assay}

Transwell invasion chamber (Corning Incorp. USA) assay was done for evaluating the invasion capacity of cells as per supplied instructions. Briefly, the chamber was rinsed priorly with MEM followed by addition of Matrigel $1 \mathrm{mg} / \mathrm{mL}(20 \mu \mathrm{L})$ for creating a Matrigel membrane by covering the membrane surface evenly. For carrying out cell invasion assay, both the cell types, i.e., RPs and ECs were incubated in serum free 
media for 12 hours and then seeded in MEM media supplemented with FBS (10\%) and transferred to the top of chamber. The lower chamber played role of chemo-attractant and contained FBS (10\%) in MEM medium. After incubating for two days, i.e., 48 hours at $25^{\circ} \mathrm{C}$, the cells from the upper chamber were harvested with the help of cotton swab, after this the invading cells were fixed in formaldehyde (4\%) for 10 minutes at $37^{\circ} \mathrm{C}$ followed by staining with crystal violet $(0.1 \%)$ for 10 minutes. The cells were evaluated for invasion using inverted microscope and were photographed and counted with the help of ImageJ software.

\section{Statistical Analysis}

All the results are presented as mean \pm SD. Two-way ANOVA was done for performing multiple comparisons. Paired Student's $t$-test was done for studying the differences between two groups. $\mathrm{P}<0.05$ were regarded as statistically significant.

\section{Results}

\section{Expression of miR-7a and IRS-2 in}

\section{Endometrial Cells and Retinal Pericytes}

The isolated ECs and RPs from mice were submitted for qRT-PCR for expression of miR-7a and IRS-2. The results of qRT-PCR suggested that expression levels of miR-7a were significantly suppressed in ECs and RPs isolated from mice subjected to DR against the normal mice (Figure 1A). The mRNA levels of IRS-2 were found to be increased in RPs and ECs against their normal controls (Figure $1 \mathrm{~B}$ and $\mathrm{C}$ ). Western blot analysis was done to evaluate the protein levels of IRS-2, the protein levels were found to be increased significantly in both the cells, i.e., RPs and ECs compared to normal control (Figure 1D).

\section{IRS-2 is the Favorable Target of miR-7a}

It was evidenced that the expression levels of miR-7a were down-regulated in both ECs and RPs isolated from DR mice. To amplify or suppress the levels of miR-7a in RPs and ECs we used miR-7a mimics and miR-7a synthetic inhibitor respectively. Further qRT-PCR was done evaluate and confirm the efficiency of the miR-7a mimics or inhibitor, the results clearly suggested that the miR-7a mimics led to over-expression and miR-7a inhibitor suppressed the expression levels of miR-7a in ECs and RPs (Figure 2A and B). In silico bioinformatics analysis done by TargetScan predicted IRS-2 as potential target site for miR-7a, accordingly, a potential binding site of miR-7a was identified in the 3 'UTR region of the IRS- 2 gene (Figure 2C). To confirm IRS-2 as the potential target

\section{B}

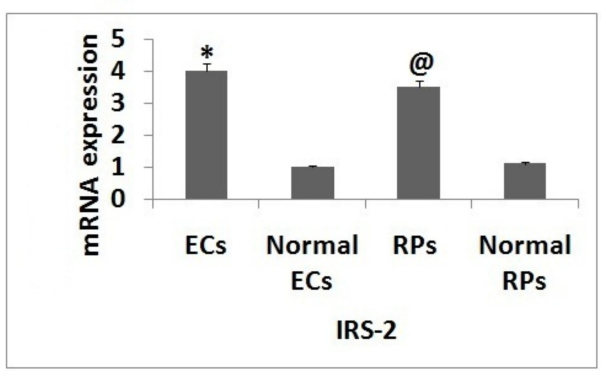

D

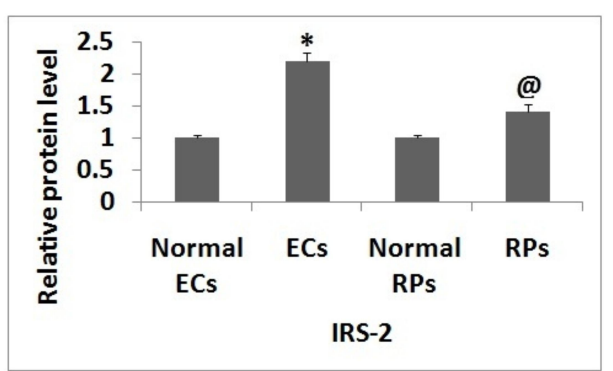

Figure I Expression of miR-7a and IRS-2 in Endothelial cells and Retinal Pericytes. (A) qRT-PCR analysis for expression levels of miR-7a in Endothelial cells and Retinal Pericytes against normal controls. (B) Results of qRT-PCR for expression of mRNA levels of IRS-2 in Endothelial cells and Retinal Pericytes against normal controls. (C) Western blot assay for protein levels of IRS-2 Endothelial cells and Retinal Pericytes. (D) Relative protein levels against Actin as loading control. The results are presented as the mean \pm standard deviation $(n=3)$. *P<0.05 compared to normal Endothelial cells; ${ }^{9} \mathrm{P}<0.05$ against normal Retinal pericytes. 
A

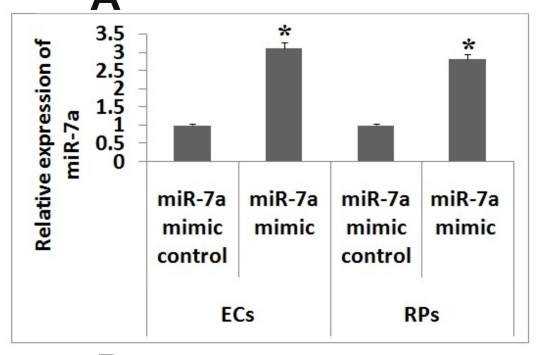

D

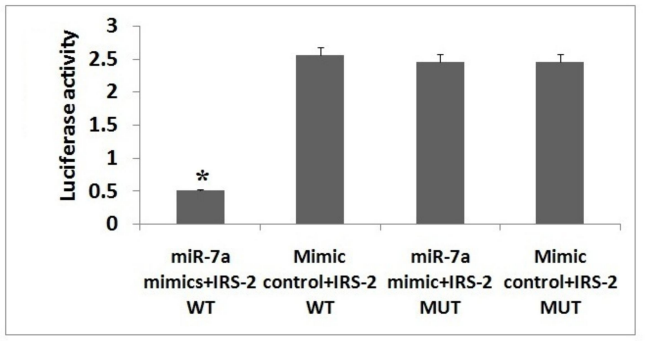

B

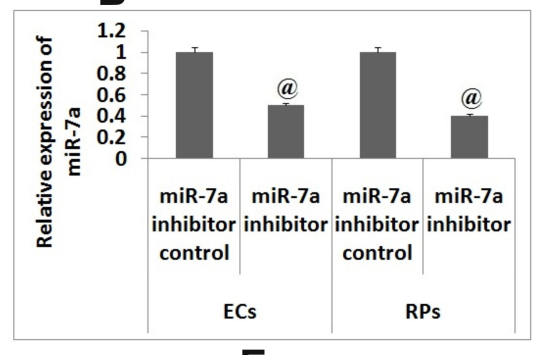

E

C

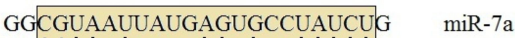
|III | || | ||||| CAGCAUCACCUAUCUCAAAUAGAA IRS-2 3'UTR

Figure 2 IRS-2 is the potential target of miR-7a. (A) qRT-PCR analysis was done for studying the levels of miR-7a in endothelial cells and Retinal pericytes co-transfected with miR-7a mimic or mimic control.(B) qRT-PCR analysis in endometrial cells and Retinal pericytes co-transfected with miR-7a inhibitor or control.(C) The IRS-2 3'UTR having potential binding site for miR-7a. (D) The Endothelial cells co-transfected with miR-7a mimic or control luciferase vector having wild type or mutant IRS-2 3'UTR. (E) Results of luciferase assay in endothelial cells transfected with miR-7a inhibitor or control, the luciferase results were normalized against renilla luciferase. Results are presented as the mean \pm standard deviation $(n=3)$. $* P<0.05$ compared to other groups, ${ }^{@} P<0.05$ compared to miR-7a mimic control.

miR-7a experimentally, the ECs were transfected with IRS-2 wild type and mutant plasmids along with miR-7a mimics or mimics controls. After 48 hours post transfection of plasmid, it was observed that the group transfected with IRS-2 wild type along with miR-7a mimic showed significant decrease in luciferase activity compared to other groups (Figure 2D). Further, the IRS-2 wild type and mutant luciferase reporter were transfected along with miR-7a inhibitor or its controls, it was found that the group transfected with miR-7a inhibitor inverted the decrease in the expression of luciferase along with IRS-2 wild type in ECs (Figure 2E). The outcome of experiment suggested IRS-2 as potential target of miR-7a.

\section{miR-7a Inhibits the Viability and Invasion Capacity of ECs and RPs}

We further studied effect of miR-7a on viability and invasion capacity of ECs and RPs. For the same the ECs and RPs were transfected with miR-7a mimic, mimic control, miR-7a inhibitor and its control. The effect of transfection on expression of IRS-2 was studied by Western blot analysis (Figure 3A and B). It was observed that when transfected with miR-7a mimic the protein levels of IRS-2 were decreased significantly as compared to mimic control and transfection of miR-7a inhibitor caused substantial increase in protein levels of IRS-2 against the control (Figure 3C). Upon transfecting ECs and RPs with
miR-7a mimic resulted in significant decrease in number of invasive cells against the mimic control group, whereas miR7a inhibitor enhanced the number of invasive RPs and ECs compared to inhibitor control (Figure 3D). Also, it was observed that overexpression of miR-7a suppressed the cell viability of ECs against the group treated with mimic control, inversely, the suppression of miR-7a increased cell viability compared to inhibitor control (Figure 3E). The outcomes of MTT assay in RPs suggested that the cell viability decreased with upregulation of miR-7a compared to that of mimic control transfected cells (Figure $3 \mathrm{~F}$ ), the viability of RPs increased after treating them with miR-7a inhibitor (Figure 3F). The findings indicated that miR-7a decreased the invasion capacity and viability of ECs and RPs.

\section{miR-7a Blocks the PI3K/Akt Cascade by Suppressing IRS-2}

VEGF is associated to maintain vascular homeostasis in ECs. ${ }^{26}$ Modulation of VEGF expression by insulin is majorly mediated via triggering of PI3K/Akt cascade. ${ }^{27}$ Hence we evaluated the outcomes of suppression and over-expression of miR-7a on levels of IRS-2 and PI3K/Akt cascade. The expression of genes such as Akt, PI3K and VEGF was studied by Western blot analysis. The outcomes suggested that the cells transfected with miR-7a mimic caused a significant suppression in protein levels of Akt, PI3K and VEGF against the 


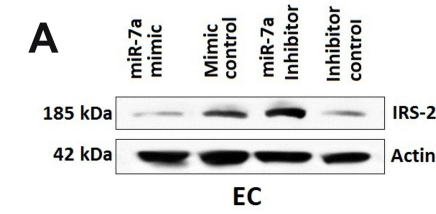

B

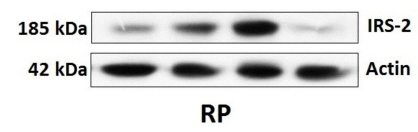

C

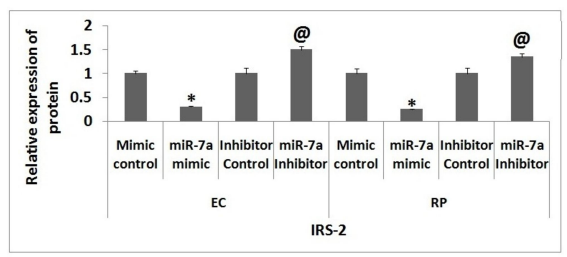

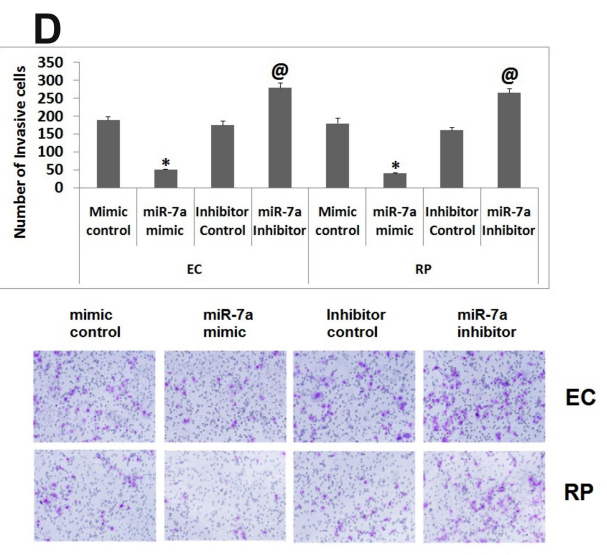
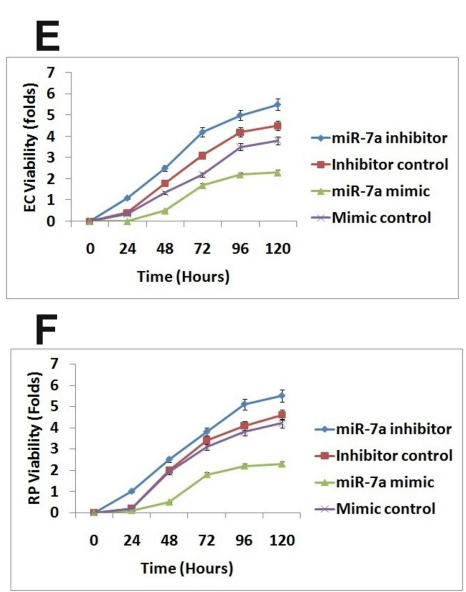

Figure 3 miR-7a inhibits the viability and invasion capacity of Endothelial cells and Retinal pericytes. (A and B) Western blot analysis for expression of IRs-2 in Endothelial cells and Retinal pericytes co-transfected with miR-7a mimic, control, miR-7a inhibitor or its control. (C) Relative expression of proteins in endothelial cells and Retinal pericytes. (D) Number of invading Endothelial cells and Retinal pericytes. (E) Results of cell viability done by MTT assay for Endothelial cells and Retinal pericytes. (F) Viability of retinal pericytes co-transfected with miR-7a mimic, control, miR-7a inhibitor or its control. Results are presented as the mean \pm standard deviation ( $\mathrm{n}=3$ ). $* \mathrm{P}<0.05$ compared to mimic control, ${ }_{\mathrm{P}}<0.05$ compared to inhibitor control.

mimic control transfected cells, whereas transfection with miR-7a inhibitor increased the protein levels of Akt, PI3K and VEGF in ECs compared to inhibitor control (Figure 4). Similarly, Western blot analysis was performed in RPs, it was observed that when transfected with miR-7a mimic resulted in decreased expression of Akt, PI3K and VEGF compared to those transfected with mimic control. Also, transfection with miR-7a inhibitor caused a significant increase in expression levels of Akt, PI3K and VEGF compared to those transfected with inhibitor control (Figure 4).The results have suggested that over-expression of miR-7a in RPs and ECs caused suppression of PI3K/Akt cascade.

\section{Intervention of IRS-2 with siRNA Neutralized the Effect of miR-7a Inhibitor in Endometrial Cells}

To study the effect of any interference with expression of IRS2 related with effects of miR-7a in ECs, the ECs along with miR-7a inhibitor or its control were co-transfected with or without a siRNA IRS-2 vector. Western blot analysis was performed for evaluating the expression of VEGF, Akt and PI3K (Figure 5A). It was observed that, upon transfection with miR-7a inhibitor resulted in significantly increased expression of VEGF, Akt and PI3K compared to group transfected with inhibitor control (Figure 5B-D). For ECs transfected with siRNA IRS-2 along with miR-7a inhibitor, the expression of
VEGF, Akt and PI3K proteins were significantly inhibited compared with those treated with miR-7a inhibitor (Figure $5 \mathrm{~B})$, whereas the protein levels were unregulated compared to siRNA IRS-2 treated group (Figure 5B-D). These findings indicated that, upon transfection of siRNA IRS-2 neutralized them in miR-7a mediated inhibition of VEGF in ECs. Further we studied the effect of miR-7a inhibitor or its control in presence and absence of siRNA IRS-2 vector on number of invading ECs, in order to confirm the effect of silencing of IRS-2. It was found that the interference of IRS-2 inverted the effect of miR-7a inhibitor on invasion property of cells (Figure 5E). In our study we also performed MTT assay to examine the ECs viability under the above mentioned treatments. We observed that cell viability increased when the ECs received co-transfection with siRNA IRS-2 and miR07a inhibitor compared to group transfected with siRNA IRS-2 (Figure 5F). These findings indicated that when IRS-2 was interfered resulted in restoration of inhibitory effect of miR-7a in ECs.

\section{Discussion}

DR is associated with development of early decrease of capillary pericytes and the associated process of angiogenesis. $^{2}$ Studies have confirmed that IRS-2 is involved in development of type- 2 diabetes. ${ }^{28}$ The findings of the present study were in agreement to this study which clearly suggested that expression of IRS-2 was overexpressed significantly in ECs and RPs isolated from 
A
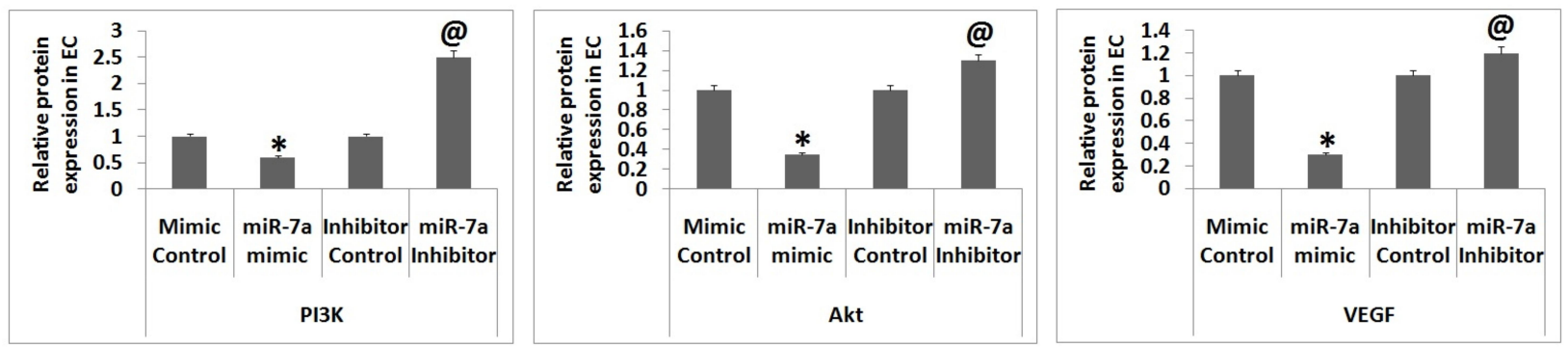

B
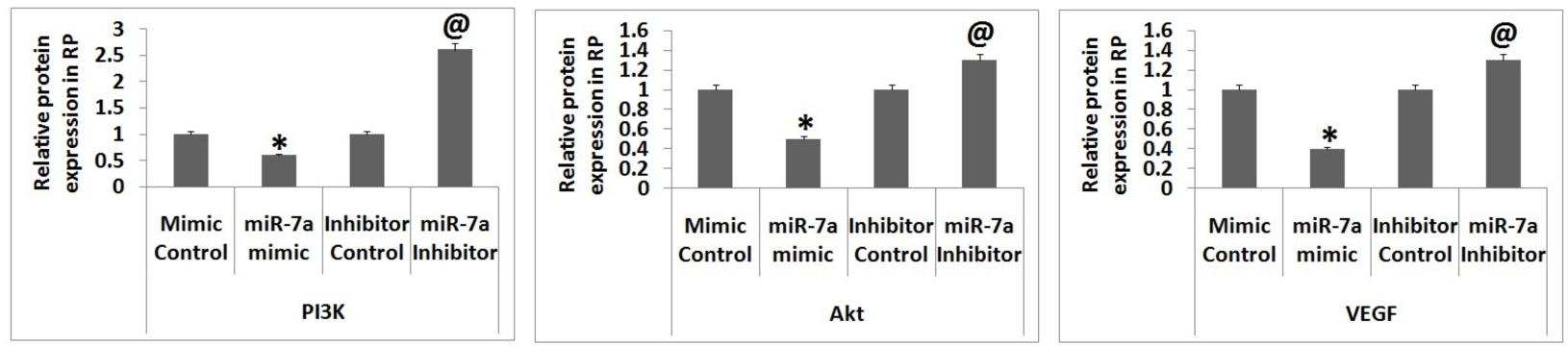

C
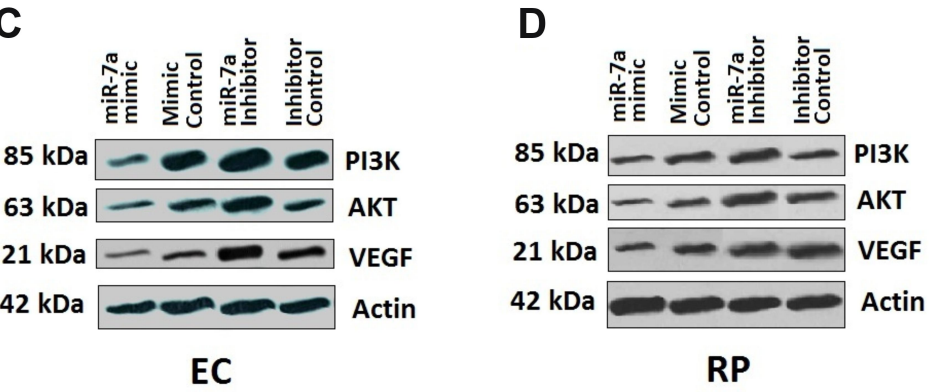

Figure 4 miR-7a regulated the PI3K/Akt cascade by targeting IRS-2. The Endothelial cells and Retinal pericytes received transfection of miR-7a mimic, mimic control, miR-7a inhibitor or inhibitor control. (A and B) Protein levels of IRS-2 and proteins of PI3K/Akt cascade. (C and D) Western blot analysis for expression of PI3K/Akt proteins in Endothelial cells and Retinal pericytes. The results are presented as mean \pm standard deviation $(n=3)$. $* \mathrm{P}<0.05$ compared to mimic control, $@_{\mathrm{P}<0.05}$ compared to inhibitor control.

mouse model of DR compared to control mice. To study the involvement of IRS-2 in DR, we performed the bioinformatics analysis and studied the 3'UTR of IRS-2. miRs are discovered to influence expression of various genes by targeting their 3'UTR region, in the present work, miR-7a was predicted to be miRNA which targets IRS-2 gene and hence may be involved in the development of DR.

miR-7a is found to regulate important functions of pancreatic biology including cell differentiation and production of insulin. $^{29}$ miR-7a is significantly overexpressed in organs such as eyes, brain, and pancreas indicating role miR-7a in development of these organs. Reports also have showed that miR-7a shows aberrant expression in breast cancer and glioma. In addition to this miR-7a targets some important genes such as SATB1, RAF1, EGFR, IRS-1. ${ }^{30,31}$ In a study miR-7a has been found to impair insulin signaling and regulates extracellular $A \beta$ levels by targeting IRS-2. ${ }^{32}$ In our study the bioinformatics analysis by TargetScan suggested that the 3'UTR region of IRS-2 gene had a potential binding site for miR-7a, hence we selected miR-7a targeting IRS-2. The outcomes of the present study suggested that expression of miR-7a was significantly inhibited when the expression of IRS-2 was increased in ECs and RPS isolated from DR mice model compared to healthy ones. The favorable target of miR-7a, i.e., IRS-2 was verified experimentally by constructing IRS-2 wild type and mutant reporter vector. The outcomes of suggested that overexpression of miR-7a blocked the luciferase activity when the ECs and RTs were transfected with IRS-2 wild type luciferase, but not in group transfected with IRS-2 mutant. Also, blockade of miR-7a enhanced the luciferase 
A
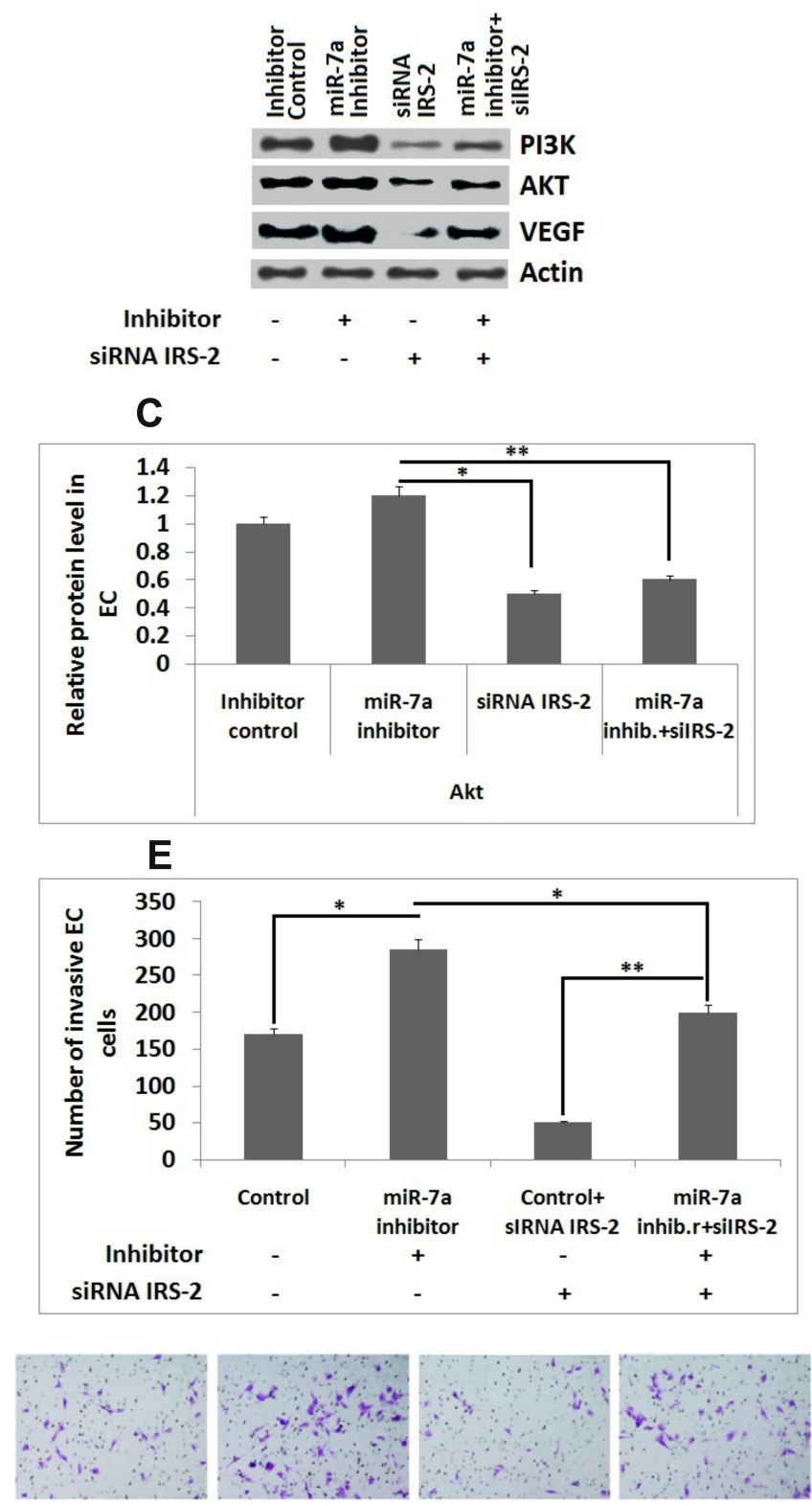

B

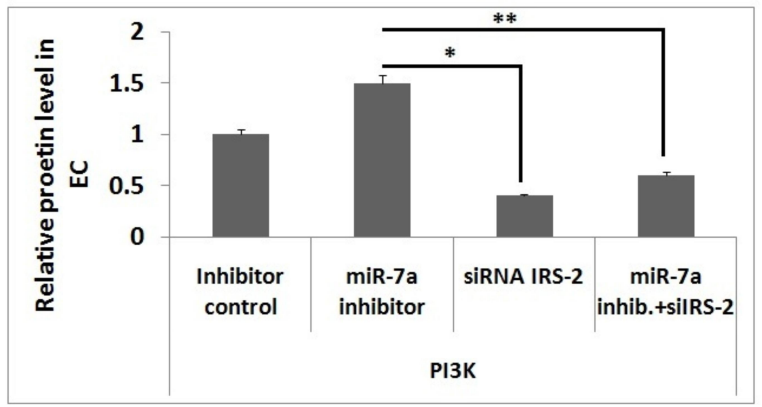

D
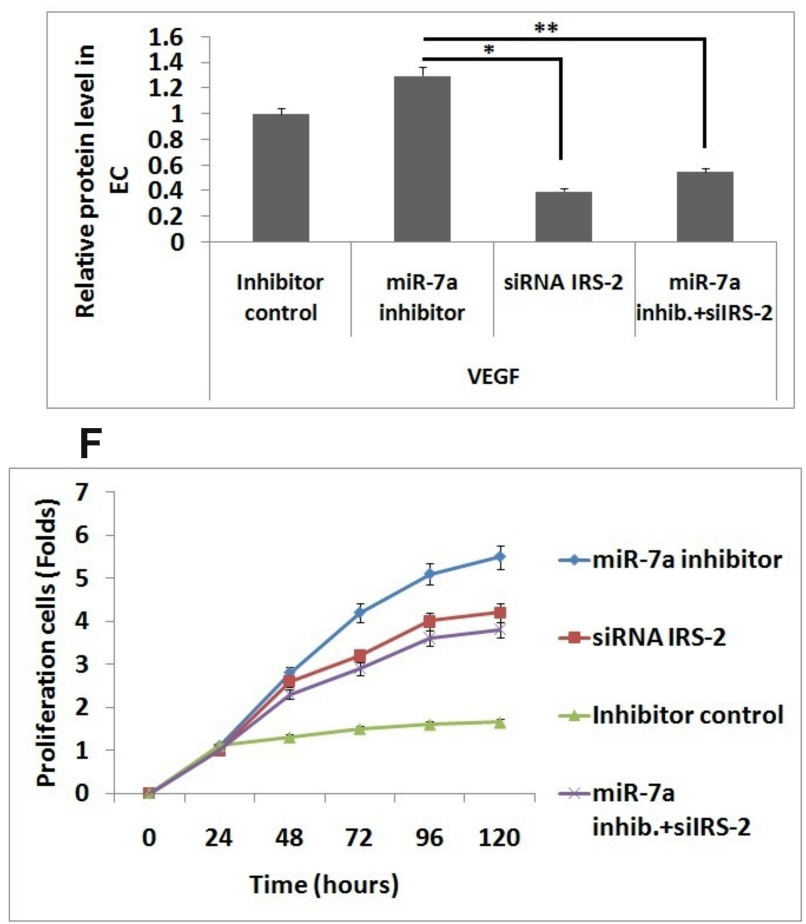

Figure 5 siRNA interferencingIRS-2 inhibits the effect of miR-7a in endothelial cells. (A) Western blot analysis for expression of PI3K, Akt and VEGF. (B-D) Relative protein levels in Endothelial cells against actin. (E) Number of invading Endothelial cells. (F) Results of MTT assay showing viability of endothelial cells. The results are mean \pm standard deviation $(\mathrm{n}=3)$. $* \mathrm{P}<0.05$ and $* * \mathrm{P}<0.01$ compared to indicated treated groups.

activity in IRS-2 wild type transfected cells against the IRS-2 mutant treated cells. The outcomes suggested that IRS-2 was favorable target gene of miR-7a in RPs and ECs isolated from DR mice. Further, we explored the involvement of miR-7a in invasion capacity and viability of RPs and ECs via targeting IRS-2, we studied the effect of miR-7a inhibition and miR-7a over-expression on invasion and viability capacity of ECs and RPs. The outcomes suggested that upon transfection with miR-7a mimic resulted in inhibition on expression levels of IRS-2 and also suppressed the invasion capacity and viability of cells. However, inhibition of miR-7a resulted in up-regulation of IRS-2 and also increased invasion capacity and viability of RPs and ECs. As reported earlier, miR-7a has been identified to target IRS-2 and impair insulin signaling. ${ }^{32}$ These findings indicated that miR-7a negatively regulated the invasion and viability of RPs and ECs isolated from DR mice targeting IRS-2.

VEGF is one of the main regulators linked with carcinogenesis and angiogenesis. ${ }^{33}$ Insulin is reported to act 
as up-regulator of VEGF via insulin receptors. ${ }^{27}$ Regulation of VEGF by insulin is reported to be the main reason for progression of DR. ${ }^{34}$ A study earlier has also concluded that insulin up-regulates the expression of VEGF through targeting Ras/MAP and PI3K/Akt cascades. ${ }^{9}$ Also VEGF is reported to be the downstream target of PI3K/Akt pathway. ${ }^{34}$ In the current study, we found that the expression of PI3K, VEGF and Akt which are protein belonging to $\mathrm{PI} 3 \mathrm{~K} / \mathrm{Akt}$ axis were suppressed after transfecting with miR-7a mimic against the control transfected group of cells. The outcomes suggested that up-regulation of miR-7a blocked the PI3K/Akt cascade proteins by suppressing the IRS-2. Insulin is evidenced to trigger various signaling pathways in ECs via activation of insulin receptors through phosphorylating the IRSs, resulting in activation of Akt. ${ }^{15} \mathrm{We}$ in our study confirmed the inhibitory action of miR-7a on expression of proteins of PI3K/Akt cascade and also on invasion capability and viability of ECs by targeting the IRS-2 gene. For the same we neutralized the IRS-2 gene with the help of siRNA in ECs. The findings clearly suggested that upon transfecting with siRNA IRS-2 fixed the suppressive effect of miR-7a on expression of proteins of PI3K/ Akt cascade in ECs. These findings indicated that miR-7a inhibits the expression of IRS-2 in RPs and ECs. These findings demonstrated the IRS-2 is novel gene associated with DR. However more studies are needed for clearly understanding the therapeutic strategy in treating DR targeting IRS-2 as target gene.

All together, the findings of the present work suggested that up-regulation of miR-7a influenced the expression of IRS-2, causing suppression VEGF proteins and also decreased the invasion capacity and viability of RPs and ECs isolated from DR mice. However more detailed study about the effect of miR-7a on other pathways involved in pathogenesis of DR need to be studied. The work also confirms the pathogenesis of DR and role of miR-7a as a favorable therapeutic candidate for DR.

\section{Data Sharing Statement}

All the data is presented in paper.The supporting study data for work is under ethics laws of university and is hence not presented here.

\section{Ethics Approval}

The animal studies were approved by the animal ethical review board of Renmin Hospital of Wuhan University, Wuhan, Hubei, China.

\section{Consent to Publish}

Hereby we declare that our institutes are aware of the work and declare consent for publication of the manuscript.

\section{Acknowledgments}

The authors are thankful to The Renmin Hospital of Wuhan University, Wuhan, Hubei, China and our funding agency National Natural Science Foundation of China.

\section{Author Contributions}

ZJ and YS contributed equally to the work. JL, TS,CC, ZJ and YS planned the study together. ZJ and YS performed the animal studies, JL, TS and CC performed the remaining experiments. All the authors prepared the manuscript and reviewed it. All authors made substantial contributions to conception and design, acquisition of data, or analysis and interpretation of data; took part in drafting the article or revising it critically for important intellectual content; agreed to submit to the current journal; gave final approval of the version to be published; and agree to be accountable for all aspects of the work.

\section{Funding}

This study was supported by National Natural Science Foundation of China (No: 81500744).

\section{Disclosure}

The authors declare no conflicts of interest for this work.

\section{References}

1. Congdon NG, Friedman DS, Lietman T. Important causes of visual impairment in the world today. JAMA. 2003;290(15):2057-2060. doi:10.1001/jama.290.15.2057

2. Armulik A, Abramsson A, Betsholtz C. Endothelial/pericyte interactions. Circ Res. 2005;97(6):512-523. doi:10.1161/01. RES.0000182903.16652.d7

3. Caldwell RB, Bartoli M, Behzadian MA, et al. Vascular endothelial growth factor and diabetic retinopathy: pathophysiological mechanisms and treatment perspectives. Diabetes Metab Res Rev. 2003;19 (6):442-455. doi:10.1002/dmrr.415

4. Saltiel AR, Pessin JE. Insulin signaling pathways in time and space. Trends Cell Biol. 2002;12(2):65-71. doi:10.1016/S0962-8924(01) 02207-3

5. White MF. IRS proteins and the common path to diabetes. $A m$ J Physiol Endocrinol Metab. 2002;283(3):413-422. doi:10.1152/ ajpendo.00514.2001

6. Ferrara N. Role of vascular endothelial growth factor in regulation of physiological angiogenesis. Am J Physiol Cell Physiol. 2001;280(6): C1358-C1366. doi:10.1152/ajpcell.2001.280.6.C1358

7. Behl T, Kotwani A. Exploring the various aspects of the pathological role of vascular endothelial growth factor (VEGF) in diabetic retinopathy. Pharmacol Res. 2015;99:137-148. doi:10.1016/j. phrs.2015.05.013 
8. Poulaki V, Qin W, Joussen AM, et al. Acute intensive insulin therapy exacerbates diabetic blood-retinal barrier breakdown via hypoxia-inducible factor-1alpha and VEGF. J Clin Invest. 2002;109 (6):805-815. doi:10.1172/JCI0213776

9. Jiang ZY, He Z, King BL, et al. Characterization of multiple signaling pathways of insulin in the regulation of vascular endothelial growth factor expression in vascular cells and angiogenesis. J Biol Chem. 2003;278(34):31964-31971. doi:10.1074/jbc.M303314200

10. American Diabetes Association. Peripheral arterial disease in people with diabetes. Diabetes Care. 2003;26(12):3333-3341. doi:10.2337/ diacare.26.12.3333

11. Waltenberger J. Impaired collateral vessel development in diabetes: potential cellular mechanisms and therapeutic implications. Cardiovasc Res. 2001;49(3):554-560. doi:10.1016/S0008-6363(00)00228-5

12. Carlsson PO, Jansson L. Disruption of insulin receptor signaling in endothelial cells shows the central role of an intact islet blood flow for in vivo $\beta$-cell function. Diabetes. 2015;64(3):700-702. doi:10.2337/db14-1523

13. Tardif K, Hertig V, Dumais C, et al. Nestin downregulation in rat vascular smooth muscle cells represents an early marker of vascular disease in experimental type I diabetes. Cardiovasc Diabetol. 2014;13(1):119. doi:10.1186/s12933-014-0119-6

14. Park SW, Yun JH, Kim JH, Kim KW, Cho CH, Kim JH. Angiopoietin 2 induces pericyte apoptosis via $\alpha 3 \beta 1$ integrin signaling in diabetic retinopathy. Diabetes. 2014;63(9):3057-3068. doi:10.2337/db13-1942

15. Jiang ZY, Lin YW, Clemont A, et al. Characterization of selective resistance to insulin signaling in the vasculature of obese Zucker ( $\mathrm{fa} /$ fa) rats. J Clin Invest. 1999;104(4):447-457. doi:10.1172/JCI5971

16. Bartel DP. MicroRNAs: target recognition and regulatory functions. Cell. 2009;136(2):215-233. doi:10.1016/j.cell.2009.01.002

17. Chen WQ, Hu L, Chen GX, Deng HX. Role of microRNA-7 in digestive system malignancy. World J Gastrointest Oncol. 2016;8 (1):121-127. doi:10.4251/wjgo.v8.i1.121

18. Li YZ, Wen L, Wei X, et al. Inhibition of miR-7 promotes angiogenesis in human umbilical vein endothelial cells by upregulating VEGF via KLF4. Oncol Rep. 2016;36(3):1569-1575. doi:10.3892/ or.2016.4912

19. Ye T, Yang M, Wang HD. MicroRNA-7 as a potential therapeutic target for aberrant NF- $\kappa \mathrm{B}$-driven distant metastasis of gastric cancer. J Exp Clin Cancer Res.2019;38(1):55. doi:10.1186/s13046-0191074-6

20. Bandello F, Lattanzio R, Zucchiatti I, Del Turco C. Pathophysiology and treatment of diabetic retinopathy. Acta Diabetol. 2013;50 (1):1-20. doi:10.1007/s00592-012-0449-3

21. Suto T, Yokobori T, Yajima R, et al. MicroRNA-7 expression in colorectal cancer is associated with poor prognosis and regulates cetuximab sensitivity via EGFRregulation. Carcinogenesis. 2015;36 (3):338-345. doi:10.1093/carcin/bgu242
22. Li Y, Li Y, Liu Y, Xie P, Li F, Li G. PAX6, a novel target of microRNA-7, promotes cellular proliferation and invasion in human colorectal cancer cells. Dig Dis Sci. 2014;59(3):598-606. doi:10.1007/s10620-013-2929-x

23. Su X, Sorenson CM, Sheibani N. Isolation and characterization of murine retinal endothelial cells. Mol Vis. 2003;9:171-178.

24. Buzney SM, Massicotte SJ, Hetu N, Zetter BR. Retinal vascular endothelial cells and pericytes. Differential growth characteristics in vitro. Invest Ophthalmol Vis Sci. 1983;24(4):470-480.

25. Zhang J, Du YY, Lin YF, Chen YT, Yang L, Wang HJ. The cell growth suppressor, mir-126, targets IRS-1. Biochem Biophys Res Commun. 2008;377(1):136-140. doi:10.1016/j.bbrc.2008.09.089

26. Hoeppner LH, Sinha S, Wang Y, et al. RhoC maintains vascular homeostasis by regulating VEGF-induced signaling in endothelial cells. J Cell Sci. 2015;128(19):3556-3568. doi:10.1242/jcs.167601

27. King GL, Goodman AD, Buzney S, Moses A, Kahn CR. Receptors and growth-promoting effects of insulin and insulinlike growth factors on cells from bovine retinal capillaries and aorta. J Clin Invest. 1985;75(3):1028-1036. doi:10.1172/JCI111764

28. Withers DJ, Gutierrez JS, Towery H, et al. Disruption of IRS-2 causes type 2 diabetes in mice. Nature. 1998;391(6670):900-904. doi: $10.1038 / 36116$

29. Latreille M, Hausser J, Stützer I, et al. MicroRNA-7a regulates pancreatic $\beta$ cell function. J Clin Invest. 2014;124(6):2722-2735. doi:10.1172/JCI73066

30. Saydam O, Senol O, Würdinger T, et al. miRNA-7 attenuation in Schwannoma tumors stimulates growth by upregulating three oncogenic signaling pathways. Cancer Res. 2011;71(3):852-861. doi:10.1158/0008-5472.CAN-10-1219

31. Giles KM, Barker A, Zhang PM, Epis MR, Leedman PJ. MicroRNA regulation of growth factor receptor signaling in human cancer cells. Methods Mol Biol. 2011;676:147-163.

32. Fernández-de Frutos M, Galán-Chilet I, Goedeke L, et al. MicroRNA 7 impairs insulin signaling and regulates $A \beta$ levels through posttranscriptional regulation of the insulin receptor substrate 2, insulin receptor, insulin-degrading enzyme, and liver $\mathrm{X}$ receptor pathway. Mol Cell Biol. 2019;39(22):e00170-19. doi:10.1128/MCB.00170-19

33. Shibuya M, Claessonwelsh L. Signal transduction by VEGF receptors in regulation of angiogenesis and lymphangiogenesis. Exp Cell Res. 2006;312(5):549-560. doi:10.1016/j.yexcr.2005.11.012

34. Simó R, Sundstrom JM, Antonetti DA. Ocular anti-VEGF therapy for diabetic retinopathy: the role of VEGF in the pathogenesis of diabetic retinopathy. Diabetes Care. 2014;37(4):893-899. doi:10.2337/dc132002

\section{Publish your work in this journal}

Diabetes, Metabolic Syndrome and Obesity: Targets and Therapy is an international, peer-reviewed open-access journal committed to the rapid publication of the latest laboratory and clinical findings in the fields of diabetes, metabolic syndrome and obesity research. Original research, review, case reports, hypothesis formation, expert opinion and commentaries are all considered for publication. The manuscript management system is completely online and includes a very quick and fair peer-review system, which is all easy to use. Visit http://www.dovepress.com/testimonials.php to read real quotes from published authors. 